\title{
Demo Abstract: Telepresence Robot with Autonomous Navigation and Virtual Reality
}

\author{
Prakash Kurup, Kaikai Liu \\ Department of Computer Engineering \\ San Jose State University \\ San Jose, CA, USA \\ \{prakash.kurup, kaikai.liu\}@sjsu.edu
}

\begin{abstract}
Telepresence technology enables users to be virtually present in another location at the same time through video streaming. This kind of user interaction is further enhanced through mobility by driving remotely to form what is called a Telepresence robot. These innovative machines connect individuals with restricted mobility and increase social interaction, collaboration and active participation. However, operating and navigating these robots by individuals who have little knowledge and map of the remote environment is challenging. Avoiding obstacles via the narrow camera view and manual remote operation is a cumbersome task. Moreover, the users lack the sense of immersion while they are busy maneuvering via the real-time video feed and, thereby, decreasing their capability to handle different tasks. This demo presents a simultaneous mapping and autonomous driving virtual reality robot. Leveraging the $2 \mathrm{D}$ Lidar sensor, we generate two dimensional occupancy grid maps via SLAM and provide assisted navigation in reducing the onerous task of avoiding obstacles. The attitude of the robotic head with a camera is remotely controlled via the virtual reality headset. Remote users will be able to gain a visceral understanding of the environment while teleoperating the robot.
\end{abstract}

\section{INTRODUCTION}

In today's world, there are many difficult scenarios when it comes to social interactions such as parents and elders living a solitary life lacking social interaction with their loved ones. Situations where physically challenged kids are unable to attend classes and interact with other school kids. Couples living far apart due to job locations or studies, causing undue stress in the relationships. Though there are many video conferencing applications available in the market these days to communicate easily across the globe, they lack the feeling of being physically present and interacting with individuals.

\footnotetext{
${ }^{*}$ The work presented in this paper is supported in part by National Science Foundation under Grant No. CNS1637371 .

Permission to make digital or hard copies of all or part of this work for personal or classroom use is granted without fee provided that copies are not made or distributed for profit or commercial advantage and that copies bear this notice and the full citation on the first page. Copyrights for components of this work owned by others than ACM must be honored. Abstracting with credit is permitted. To copy otherwise, or republish, to post on servers or to redistribute to lists, requires prior specific permission and/or a fee. Request permissions from permissions@ acm.org.

SenSys '16 November 14-16, 2016, Stanford, CA, USA

(C) 2016 ACM. ISBN 978-1-4503-2138-9...\$15.00

DOI: $10.1 .145 / 2994551.2996540$
}

Telepresence system overcomes the above challenges and allow remote users to feel they are in the same physical space, when in reality they are in different locations in the world. Adding to mobility to the system further enhances the telepresence experience and allow the users to explore remote environments. Existing commercially available telepresence robots such as Double Robotics, VGo, and Anybot assist remote users in communicating and collaborating in office, schools, hospitals, factories and home environment. They can also be used for safety applications where the user can operate remotely in the dangerous or hazardous environment.

Due to limited visual angle of the camera on the robot, the real-time video feeds viewed on the remote user's computer screen lacks the feeling of the immersive experience. This can also affect the remote user's awareness of the environment and teleoperation of the robot. According to a series of user studies with two different commercially available robots at Google, autonomous navigation was highly desirable due to safety reasons and ease of use. Autonomous navigation benefits the remote user by reducing the cumbersome task of maneuvering the robot to the user's destination in the indoor environment.

Assisted navigation around obstacles helps remote users who are elderly or young children when operating these robots. Our method will implement Simultaneous Localization and Mapping (SLAM) algorithm with the help of laser scanner and robot pose data to create a two dimensional occupancy grid map of an unknown environment. The saved map is later used for autonomous navigation with obstacle avoidance, by passing a destination coordinate within the map. Our method implements concepts of virtual reality technology with telepresence robot using Google Cardboard VR, so remote users gain a greater field of view of the environment and enhanced feeling of immersion with the help of head tracking system [2].

\section{SYSTEM DESIGN}

As shown in Fig. 1, the hardware system consists of iRobot Create 2 as the robot base that interfaced to Raspberry Pi 3 embedded development board, which acts as the robot's brain. To enable mapping and localization capability, a lowcost laser scanner, RPLIDAR, is connected to the board via USB to Serial interface. The LiDAR is suitable for applications involving indoor and outdoor SLAM using 360-degree scan field of scanning frequency $5.5 \mathrm{~Hz}$ and laser range of 6 meters. Additionally, a camera module, attached to a pantilt servo bracket, is interfaced to the board for implementing 


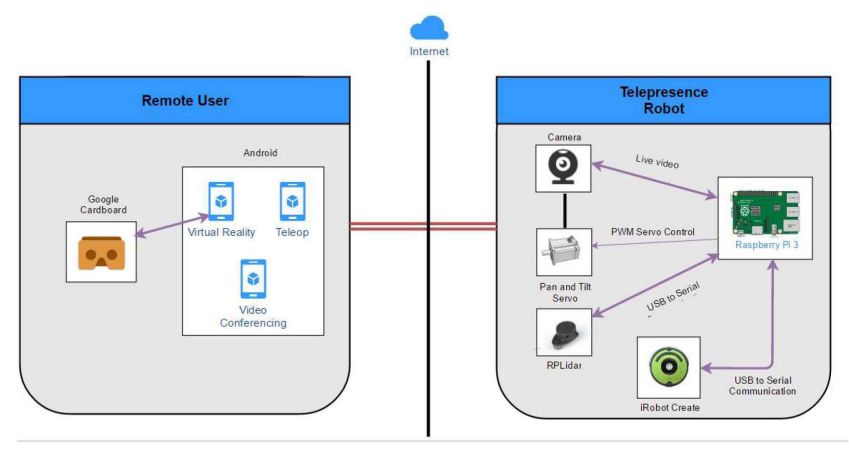

Figure 1: System Architecture.

virtual reality application.

For generating the indoor map, we use ROS wrapper application for GMapping algorithm which utilizes laser scanned data from RPLIDAR and odometry from the Create 2 base. It utilizes Rao-Blackwellized particle filters in which each particle consists of an individual map of the environment [1]. However, the parameters for the algorithm provided in the application are designed for high speed and large range sensors such as Kinect camera and are not suitable for the LiDAR specifications. We proposed a new algorithm that make the GMapping algorithm suitable for the Lidar sensing results.

The rotation direction of RPLIDAR assumes positive angle in a clockwise direction and the Z-axis of the laser scan frame must be directed to the bottom of the LiDAR. Also, the laser scan messages for minimum and maximum angles are modified to use a frame that is rotated by 180-degree for satisfying GMapping required conditions and the resulting angles equates to positive and negative $\mathrm{Pi}$ values. With the help of teleoperation using a joypad, it is possible to drive the robot and create a map of an unknown indoor environment which is visualized in the RVIZ tool running in ROS.

Once the complete indoor map is generated, it is saved as a yaml file and later exported to ROS wrapper application for Adaptive Monte Carlo Localization (AMCL) algorithm. The robot uses features in the map with laser data to identify and match features to localize itself. Each possible location is represented by a 'particle', and particles with laser scans that do not match well are removed resulting in a group of particles representing the location of the robot in the map [3]. Using RVIZ tool, the robot is initially given a hint of its approximate location within the map and then a destination point is passed for the robot to autonomously navigate while avoiding obstacles. Few experiments were conducted to verify the accuracy of the map generated and location of the objects within the map when compared to the physical world. Also, the robot's localization was examined by running autonomous navigation tests in an existing map. The coordinates generated from the robot and the objects within the map were used to calculate the distance measurements in meters and are found to be nearly accurate when compared to the physical measurements.

\section{DEMONSTRATION}

To verify the accuracy of the robot location when using autonomous navigation feature, three trash cans in one indoor environment are used as reference points for the robot

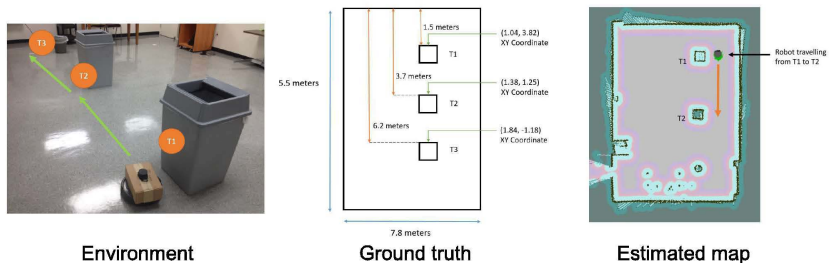

Figure 2: Experimental Results.

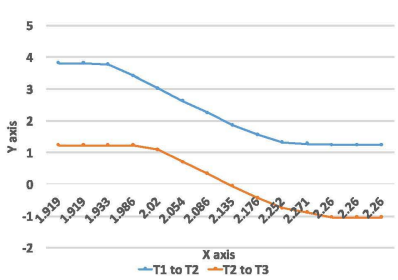

(a) Robot moving traces

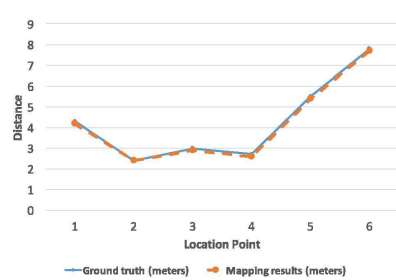

(b) Estimated distances
Figure 3: 1) The robot moving traces from $\mathrm{T} 1$ to $\mathrm{T} 2$ and T2 to T3; 2) The estimated distances vs. the ground truth distance in different locations.

to navigate from one point to another. As shown in Fig. 2, the estimated indoor map is very accurate when compared with the ground truth.

Fig. 3a shows the location traces that the robot drive autonomously from $\mathrm{T} 1$ to $\mathrm{T} 2$ and then from $\mathrm{T} 2$ to $\mathrm{T} 3$ with reference to the coordinates of $\mathrm{T} 1, \mathrm{~T} 2$ and $\mathrm{T} 3$ obtained from the map. Fig. 3b shows 6 estimated distance when compared with the ground truth. The locations are accurately estimated by the Lidar.

\section{CONCLUSION}

A video demonstrating VR control and teleoperation of the robot is available online at https://goo.gl/q7B3HD.

\section{REFERENCES}

[1] G. Grisetti, C. Stachniss, and W. Burgard. Improved techniques for grid mapping with rao-blackwellized particle filters. IEEE transactions on Robotics, 23(1):34-46, 2007.

[2] Y. Kato. A remote navigation system for a simple tele-presence robot with virtual reality. In Intelligent Robots and Systems (IROS), 2015 IEEE/RSJ International Conference on, pages 4524-4529. IEEE, 2015.

[3] B. Zhang, J. Liu, and H. Chen. Amcl based map fusion for multi-robot slam with heterogenous sensors. In Information and Automation (ICIA), 2013 IEEE International Conference on, pages 822-827. IEEE, 2013. 